\title{
ON FACTORABLE BIGRAPHIC PAIRS ${ }^{1}$
}

\author{
Jian-Hua Yin AND Sha-Sha Li \\ Department of Mathematics \\ College of Information Science and Technology \\ Hainan University, Haikou 570228, P.R. China \\ e-mail: yinjh@hainu.edu.cn
}

\begin{abstract}
Let $S=\left(a_{1}, \ldots, a_{m} ; b_{1}, \ldots, b_{n}\right)$, where $a_{1}, \ldots, a_{m}$ and $b_{1}, \ldots, b_{n}$ are two sequences of nonnegative integers. We say that $S$ is a bigraphic pair if there exists a simple bipartite graph $G$ with partite sets $\left\{x_{1}, x_{2}, \ldots, x_{m}\right\}$ and $\left\{y_{1}, y_{2}, \ldots, y_{n}\right\}$ such that $d_{G}\left(x_{i}\right)=a_{i}$ for $1 \leq i \leq m$ and $d_{G}\left(y_{j}\right)=b_{j}$ for $1 \leq j \leq n$. In this case, we say that $G$ is a realization of $S$. Analogous to Kundu's $k$-factor theorem, we show that if $\left(a_{1}, a_{2}, \ldots, a_{m} ; b_{1}, b_{2}, \ldots, b_{n}\right)$ and $\left(a_{1}-e_{1}, a_{2}-e_{2}, \ldots, a_{m}-e_{m} ; b_{1}-f_{1}, b_{2}-f_{2}, \ldots, b_{n}-f_{n}\right)$ are two bigraphic pairs satisfying $k \leq f_{i} \leq k+1,1 \leq i \leq n$ (or $k \leq e_{i} \leq k+1$, $1 \leq i \leq m$ ), for some $0 \leq k \leq m-1$ (or $0 \leq k \leq n-1)$, then $\left(a_{1}, a_{2}, \ldots, a_{m}\right.$; $\left.b_{1}, b_{2}, \ldots, b_{n}\right)$ has a realization containing an $\left(e_{1}, e_{2}, \ldots, e_{m} ; f_{1}, f_{2}, \ldots, f_{n}\right)$ factor. For $m=n$, we also give a necessary and sufficient condition for an $\left(k^{n} ; k^{n}\right)$-factorable bigraphic pair to be connected $\left(k^{n} ; k^{n}\right)$-factorable when $k \geq 2$. This implies a characterization of bigraphic pairs with a realization containing a Hamiltonian cycle.
\end{abstract}

Keywords: degree sequence, bigraphic pair, Hamiltonian cycle.

2010 Mathematics Subject Classification: 05C07.

\section{REFERENCES}

[1] J.A. Bondy and U.S.R. Murty, Graph Theory with Applications (American Elsevier, New York, 1976).

[2] Y. Chen, A short proof of Kundu's k-factor theorem, Discrete Math. 71 (1988) $177-179$. doi:10.1016/0012-365X(88)90070-2

\footnotetext{
${ }^{1}$ Supported by National Natural Science Foundation of China (Grant No. 11561017) and Hainan Provincial Natural Science Foundation of China (Grant No. 2016CXTD004).
} 
[3] D. Gale, A theorem on flows in networks, Pacific J. Math. 7 (1957) 1073-1082. doi:10.2140/pjm.1957.7.1073

[4] D.J. Kleitman and D.L. Wang, Algorithm for constructing graphs and digraphs with given valences and factors, Discrete Math. 6 (1973) 79-88. doi:10.1016/0012-365X(73)90037-X

[5] S. Kundu, The k-factor conjecture is true, Discrete Math. 6 (1973) 367-376. doi:10.1016/0012-365X(73)90068-X

[6] S. Kundu, Generalizations of the k-factor theorem, Discrete Math. 9 (1974) 173-179. doi:10.1016/0012-365X(74)90147-2

[7] A.R. Rao and S.B. Rao, On factorable degree sequences, J. Combin. Theory Ser. B 13 (1972) 185-191. doi:10.1016/0095-8956(72)90055-X

[8] H.J. Ryser, Combinatorial properties of matrices of zeros and ones, Canad. J. Math. 9 (1957) 371-377. doi:10.4153/CJM-1957-044-3

[9] J.H. Yin, A characterization for a graphic sequence to be potentially $C_{r}$-graphic, Sci. China Math. 53 (2010) 2893-2905.

doi:10.1007/s11425-010-3124-6

Received 15 September 2017

Revised 5 May 2018

Accepted 5 May 2018 